Provided for non-commercial research and education use. Not for reproduction, distribution or commercial use.

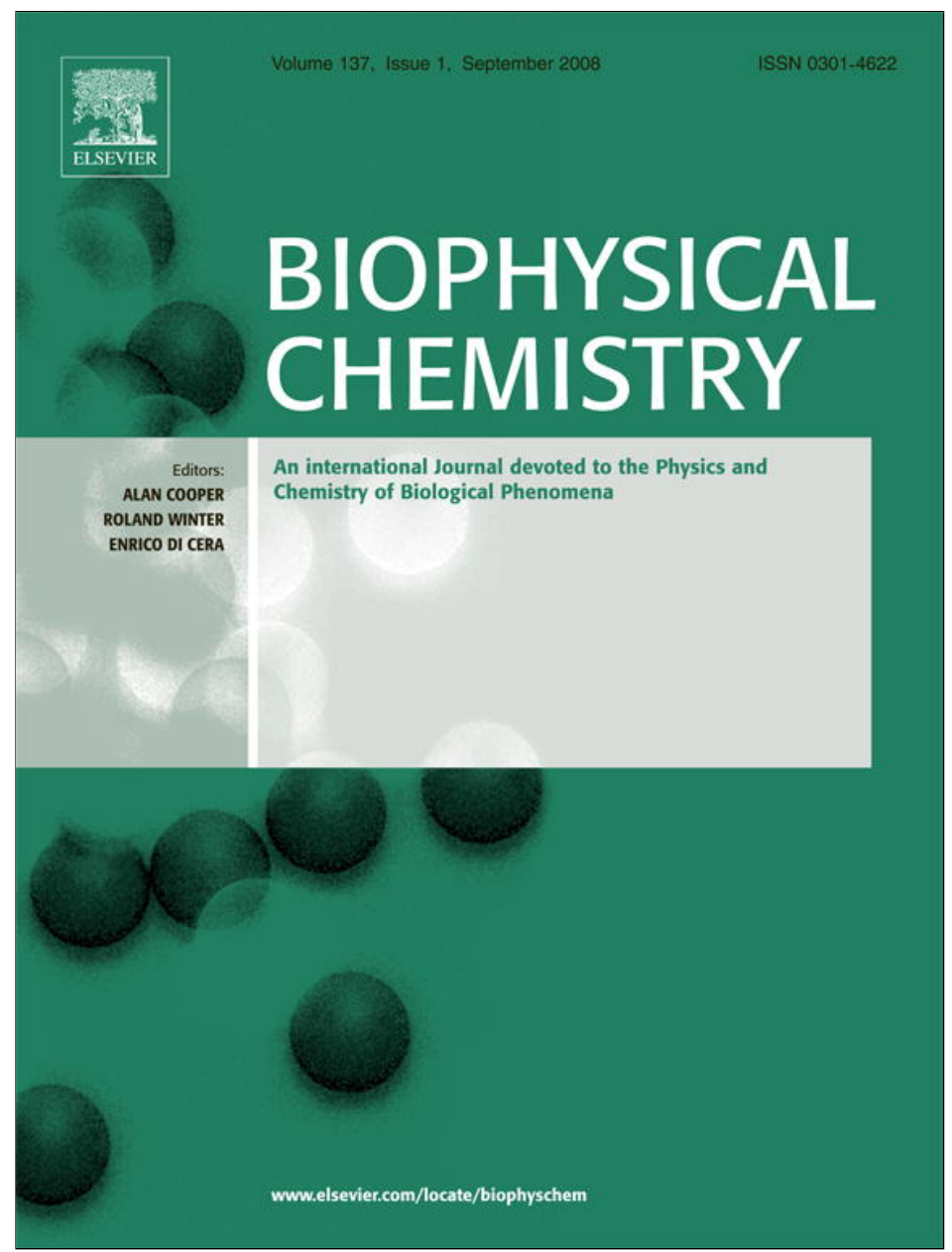

This article appeared in a journal published by Elsevier. The attached copy is furnished to the author for internal non-commercial research and education use, including for instruction at the authors institution and sharing with colleagues.

Other uses, including reproduction and distribution, or selling or licensing copies, or posting to personal, institutional or third party websites are prohibited.

In most cases authors are permitted to post their version of the article (e.g. in Word or Tex form) to their personal website or institutional repository. Authors requiring further information regarding Elsevier's archiving and manuscript policies are encouraged to visit:

http://www.elsevier.com/copyright 


\title{
Protein-film voltammetry: A theoretical study of the temperature effect using square-wave voltammetry
}

\author{
Rubin Gulaboski ${ }^{\mathrm{a}, \mathrm{c}, *}$, Milivoj Lovrić $^{\mathrm{b}}$, Valentin Mirčeski ${ }^{\mathrm{c}}$, Ivan Bogeski ${ }^{\mathrm{a}}$, Markus Hoth ${ }^{\mathrm{a}}$ \\ a Department of Biophysics, Saarland University, Homburg, Germany \\ ${ }^{\mathrm{b}}$ Rudjer Boskovic Institute, Zagreb, Croatia \\ ${ }^{c}$ Institute of Chemistry, Faculty of Natural Sciences and Mathematics, Skopje, Republic of Macedonia
}

\section{A R T I C L E I N F O}

\section{Article history:}

Received 13 June 2008

Received in revised form 30 June 2008

Accepted 30 June 2008

Available online 8 July 2008

\section{Keywords:}

Square-wave voltammetry

Temperature effect

Protein-film voltammetry

Kinetic characterization

Thermodynamic parameters

Activation energy

\begin{abstract}
A B S T R A C T
Square-wave voltammetry of surface redox reactions is considered as an adequate model for a protein-film voltammetric setup. Here we develop a theoretical approach to analyze the effects of temperature on squarewave voltammograms. The performed simulations address the surface redox reactions featuring slow, modest and fast electron transfer. The theoretical calculations show that the temperature affects the squarewave voltammetric responses in a complex way resulting in a variety of peak shapes. Temperature effects on the phenomena known as "quasireversible maximum" and "split SW peaks" are also analyzed. The simulated results can be used to analyze the redox mechanisms and kinetic parameters of electron transfer reactions in protein-film criovoltammetry and other surface-confined redox systems. Our analysis also shows how "abnormal" features present in some square-wave voltammetric studies can easily be misinterpreted by postulating "multiple species", "stable radicals", or additional processes. Finally we provide a simple algorithm to use the "quasireversible maximum" to determine the activation energy of electron transfer reactions by surface redox systems.
\end{abstract}

(C) 2008 Elsevier B.V. All rights reserved.

\section{Introduction}

Modern voltammetric techniques have tremendous practical applications in biological, pharmaceutical and environmental chemistry $[1,2]$. The voltammetric techniques have successfully been used to develop various methodologies for quantifying and studying the mechanistic pathways of numerous important bioactive compounds in artificial and biological matrixes [3,4]. Application of voltammetry for probing the chemistry of redox proteins has recently emerged as an especially simple and powerful method of investigating biologically relevant redox-active compounds [5-8]. By simple adsorption of the redox protein sample onto the surface of some suitable lipophilic electrode, insights into the processes of electron transfer and proteinprotein interactions can be obtained from experiments performed in common voltammetric setup [5-8]. Very often, the protein-film voltammetry is performed with fast-scan rates, which allows to get access to the coupled reactions taking place in the system even in the sub-millisecond timescale. Within the last 10 years, low temperature voltammetry became a popular technique for various purposes. Studying the electrochemical processes at lowered temperatures is

\footnotetext{
* Corresponding author. Institut für Biophysik, Gebäude 58, Universität des Saarlandes, D-66421 Homburg/Saar, Germany. Tel.: +49 6841 1626452; fax: +49 6841 1626060.

E-mail address: rubingulaboski@excite.com (R. Gulaboski).
}

important for a better understanding of the mechanistic pathways of many systems $[6,9]$. Low temperature voltammetry has also been a very useful technique for studying thermally unstable species, detecting electrochemically produced intermediates, and probing the redox reactions in solvents with very low polarity [9-13]. The possibility of studying the electrochemical features of different electrolytes at lowered temperatures attracts also huge attention in the fields of lithium-ion batteries $[2,14]$. The low temperature voltammetry is currently seen as a powerful alternative to fast-scan protein-film voltammetry [6,15-17]. By performing experiments in partially organic solvent mixtures at temperatures lower than $-90^{\circ} \mathrm{C}$, one can slow down the chemical reactions associated with electron transfer at the protein redox active sites and study these reactions using slower scan-rates [9]. The behaviour of the systems studied in this way mimics that observed in fast-scan experiments at room temperature.

We present theoretical results about the behaviour of the squarewave voltammograms of a simple surface redox reaction as a function of the temperature. Square-wave voltammetry was chosen, since it is the most advanced and most sophisticated technique of the pulse voltammetric techniques [2]. The surface redox reactions are regarded as an adequate model for protein-film voltammetric experiments, in which the redox active proteins and the products of their electrochemical transformation are strongly adsorbed on the surface of the working electrode. To the best of our knowledge, there is no theoretical study considering the effect of temperature under voltammetric conditions. Our simulations should help to better 
understand the redox mechanisms, kinetics and thermodynamic parameters of electron transfer reactions in protein-film criovoltammetry and other surface-confined redox systems.

\section{Mathematical model and simulation details}

The considered surface redox system in this work is described by the following reaction scheme:

$A_{(\mathrm{ads})}+n e^{-} \Leftrightarrow B_{(\mathrm{ads})}$

in which the charge of the species is omitted. It is assumed that all participants of the reaction are irreversibly immobilized (adsorbed) on the electrode surface. During the voltammetric experiment the mass transport of all species is neglected. The electrode mechanism is mathematically represented by the following set of equations

$\mathrm{d} \Gamma(A) / \mathrm{d} t=-I /(n F S)$

$\mathrm{d} \Gamma(B) / \mathrm{d} t=I /(n F S)$

$t=0 ; \Gamma(A)=\Gamma^{*} ; \Gamma(B)=0$

$t>0 ; \Gamma(A)+\Gamma(B)=\Gamma^{*}$

$\Gamma$ is a symbol of the surface concentration of particular specie that is a function of time t. $\Gamma^{*}$ is the total surface concentration of all species. $I$ is the symbol of the current, $S$ is the electrode surface area, $F$ is the Faraday constant, while $n$ is a number of exchanged electrons in an elementary act of electrochemical transformation. The solutions for the surface concentrations of the electroactive species A and B are:

$\Gamma(\mathrm{A})=\Gamma^{*}-\int_{0}^{t} \frac{I}{n F S} d \tau$

$\Gamma(\mathrm{B})=\int_{0}^{t} \frac{I}{n F S} \mathrm{~d} \tau$

Considering the Butler-Volmer formalism, at the electrode surface the following condition applies:

$\frac{I}{n F S}=k_{S} \exp (-\alpha \phi)[\Gamma(\mathrm{A})-\exp (\phi) \Gamma(\mathrm{B})]$

where $k_{\mathrm{s}}\left(\mathrm{s}^{-1}\right)$ is the heterogeneous electron exchange rate constant corresponding to the standard redox potential $E_{\mathrm{A} / \mathrm{B}}^{\theta}$ of the electrode reaction, $\alpha$ is the cathodic electron transfer coefficient, and $\phi=$ $\frac{n F}{R T}\left(E-E_{\mathrm{A} / \mathrm{B}}^{\theta}\right)$ is the dimensionless relative electrode potential. According to the simple transition state theory, the standard rate constant can be defined as: $k_{\mathrm{s}}=\operatorname{Aexp}\left(-E_{\mathrm{a}}(\mathrm{RT})^{-1}\right)$, where $A$ is the frequency factor and $E_{\mathrm{a}}$ is the activation free energy of activated complex [3]. Substituting Eqs. (5) and (6) into the Eq. (7) yields:

$\frac{I}{n F S}=k_{S} \exp (-\alpha \phi)\left[\Gamma(\mathrm{A}) *-\int_{0}^{t} \frac{I}{n F S} \mathrm{~d} \tau-\exp (\phi) \int_{0}^{t} \frac{I}{n F S} d \tau\right]$

Integral Eq. (8) is a general mathematical solution of the simple surface electrode mechanism. Numerical solution of the Eq. (8) adopted for SWV was obtained according to the method of Nicholson and Olmstead [18]. For numerical solution the time increment $d$ was defined as $d=1 /(50 f)$, where $f$ is the frequency of the potential modulation. It means that each SW half-period $\tau / 2$ was divided into 25 increments. The numerical solution reads:

$\Psi_{m}=\frac{K e^{-\alpha \varphi_{m}}\left[1-\frac{1+e^{\varphi_{m}}}{50} \sum_{j=1}^{m-1} \Psi_{j}\right]}{1+\frac{K e^{-\alpha \varphi_{m}}}{50}\left[1+e^{\varphi_{m}}\right]}$.

Here, $K$ is the dimensionless electrode kinetic parameter, defined as $K=A f^{-1} \exp \left(-E_{\mathrm{a}}(\mathrm{RT})^{-1}\right)$, while $\Psi$ is the dimensionless current defined as $\Psi=\frac{I}{n F S \Gamma^{*} F^{*}}$

Theoretical net SW voltammograms are bell-shaped curves characterized by peak potential $\Delta E_{\mathrm{p}}$, peak current $\Psi_{\mathrm{p}}$, and half-peak width $\Delta E_{\mathrm{p} / 2}$. By $\Psi_{\text {red }}$ and $\Psi_{\text {ox }}$ we assign the cathodic (reduction) and anodic (oxidation) currents of the voltammograms, respectively. All these parameters of the voltammetric curves are mainly dependent on the potential modulation parameters (frequency- $f$, amplitude $-E_{\mathrm{sw}}$, and potential increment $\Delta E$ ), as well as on the dimensionless redox kinetic parameter $K$, the number of exchanged electrons $n$, the electron transfer coefficient $\alpha$, and the temperature $T$. Detailed studies of the features of simple surface redox reaction as a function of the kinetic parameter $K, E_{\mathrm{sw}}, \alpha$, and $n$ under conditions of square-wave voltammetry can be found elsewhere $[2,19,20]$. In this communication we only focus on the influence of the temperature to the main attributes of the square-wave voltammograms of a simple surface redox reaction. All the simulations have been performed with help of the MATHCAD software.

From Eq. (9) follows that temperature affects the voltammetric response through two parameters, i.e., the relative dimensionless potential $\phi$ and the electrode kinetic parameter $K$. By varying the temperature, the two mentioned parameters are simultaneously altered. To understand the influence of each parameter separately, in the first set of simulation results presented in the following subsections 3.1 to 3.3 (Figs. 1-7), it is assumed that the electrode kinetic parameter $K$ is constant, and the temperature affects only the parameter $\phi$. This type of simulations corresponds to a comparison of
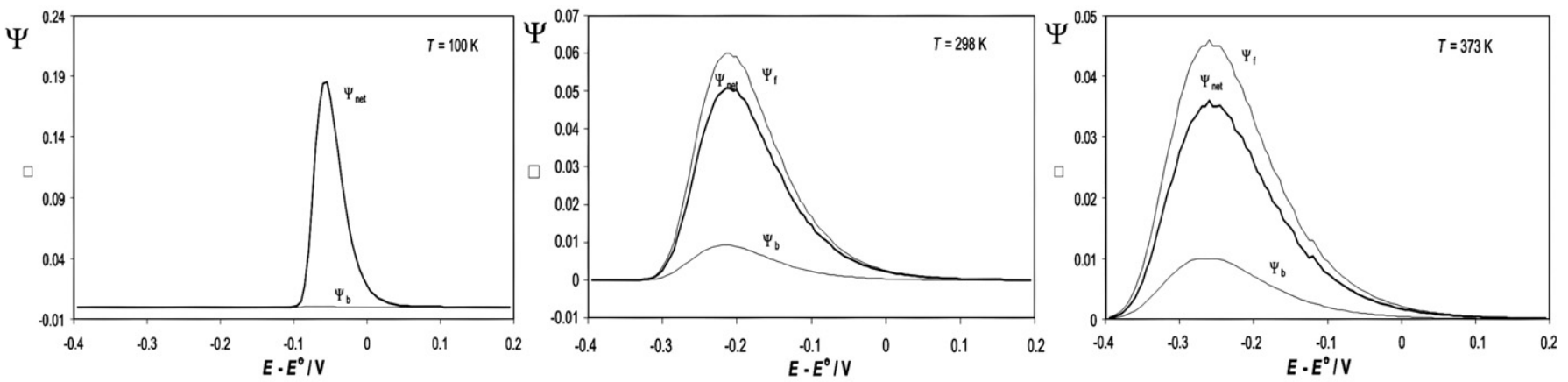

Fig. 1. Slow electron transfer: square-wave voltammograms simulated for different temperatures. The simulation parameters are: dimensionless kinetic parameter $K=0.001$, squarewave amplitude $E_{\mathrm{sw}}=50 \mathrm{mV}$; potential increment $\Delta E=2 \mathrm{mV}$, number of exchanged electrons $n=1$, and electron transfer coefficient $\alpha=0.5$. 


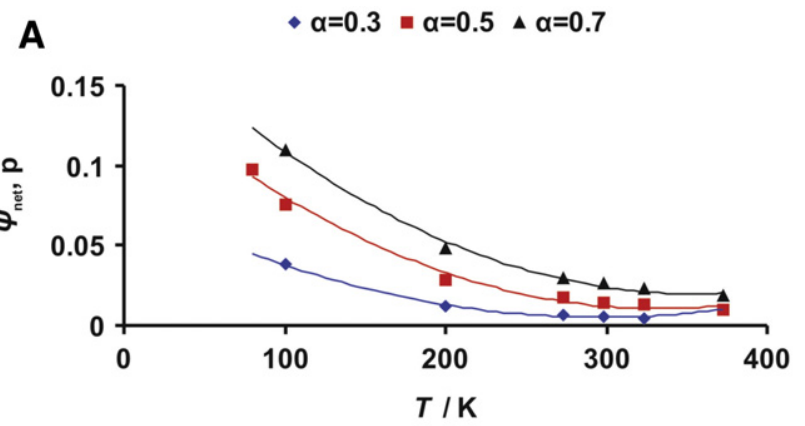

B

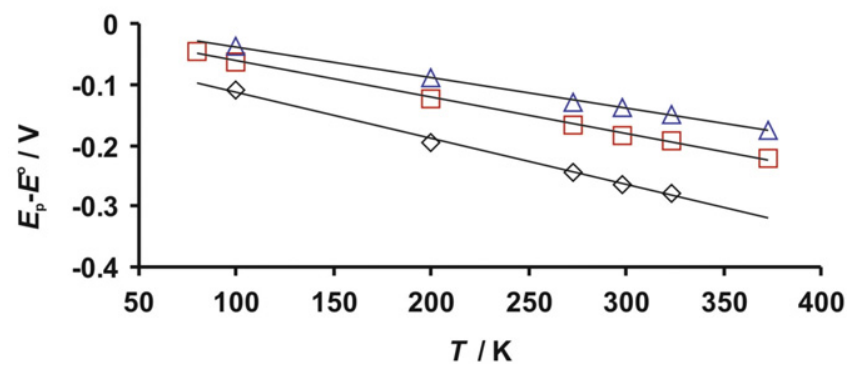

Fig. 2. Slow electron transfer: A) The peak currents and B) the peak potentials temperature dependence of the theoretical square-wave voltammograms are shown. The simulation details are the same as those in Fig. 1.

different experimental systems, which have identical electrode kinetic parameters at a particular temperature. The results presented in the subsection 3.4 correspond to the analysis of a single surface electrode reaction, during which the temperature influences the two parameters $\phi$ and $K$ simultaneously.

\section{Results and discussion}

\subsection{Irreversible electron transfer}

In the region of very sluggish (irreversible) electron transfer, i.e., in case the value of the dimensionless kinetic parameter is $\log (K)<-2$, all the features of the theoretical square-wave voltammograms of a simple surface redox reaction are strongly affected by the temperature (see Fig. 1). Evidently, for a given square-wave amplitude $E_{\mathrm{sw}}$ and electron transfer coefficient $\alpha$, decreasing the temperature increases the electrochemical reversibility of the simulated voltammograms. This effect is represented in augmentation of the magnitudes of both oxidation and reduction current components. In the same time, lowering of the temperature produces an effect to the shape of the backward current component that starts getting a form and sign typical for quasireversible redox systems.

The net peak currents of the theoretical voltammograms increase exponentially by decreasing of $T$ (see Fig. $2 \mathrm{~A}$ ). As the temperature is incorporated in the exponential term of the current-potential interdependence of the Butler-Volmer equation applied to the considered system (see Eq. (7)), this feature was expected. Besides, the net peak potentials of theoretical SW voltammograms $\left(\Delta E_{\mathrm{p}}\right)$ shift linearly in negative direction by increasing of the temperature (see Fig. 2B). The linear dependence between $\Delta E_{\mathrm{p}}$ and $T$ is represented as $\Delta E_{\mathrm{p}}=\left(-c \frac{R}{\alpha n F}\right) T$, where the constant $c$ in the slope is a function of the square-wave amplitude, while $R, n$ and $F$ are the universal gas constant, number of the exchanged electrons, and the Faraday constant, respectively.

Another very relevant parameter of square-wave voltammograms is

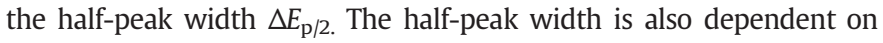
temperature in case of very slow electron transfer as shown in Fig. 3. $\Delta E_{\mathrm{p} / 2}$ decreases linearly with decreasing temperature, with a slope being inversely proportional of $\alpha$. The dependence $\Delta E_{\mathrm{p} / 2} v s$. $T$ in the irreversible region is given as $\Delta E_{\mathrm{p} / 2}=\left(z \frac{R}{\alpha n F}\right) T$, where the factor $z$ is a constant depending on $E_{\mathrm{sw}}$. A very important result in this set of simulations is the independence of the half-peak width $\Delta E_{\mathrm{p} / 2}$ on the kinetic parameter $K$ $\left(K=k_{s} / f\right)$, in the regions of $K \leq 0.005$ [2]. This feature allows a very easy calculation of $\alpha$. If we bring the redox reaction in the irreversible region (by increasing of the $S W$ frequency), and if $\Delta E_{\mathrm{p} / 2}$ is analyzed as function of $T$, it is possible to estimate the value of electron transfer coefficient $\alpha$ through comparison of the experimental slope with the theoretical ones shown in Fig. 3.

\subsection{Quasireversible electron transfer}

In case the value of the dimensionless kinetic parameter $K$ falls within interval $-2<\log (K)<0.5$, the reaction is in the region of "quasireversible electron transfer" [2,19,21]. The effect of the temperature on theoretical square-wave voltammograms in the quasireversible region is presented in Fig. 4. Simulations show that one distinctive attribute in this region is the very slight temperature dependence of the SW net peak potentials. This is basically the opposite behaviour to that observed in the region of irreversible electron transfer. The temperature, however, has a big influence to the shape of the oxidation and reduction components of the SW voltammograms; the widths of them are getting narrower if lowering the temperature. At very low temperatures, the net peak of the SW voltammogram even splits up in two peaks. The splitting of the net peak is usually caused by the skew of forward and backward current components on the potential scale, as the value of the dimensionless kinetic parameter $K$ increases $[2,19]$. The splitting effect is a feature of the surface redox reactions exhibiting very fast electron transfer. This phenomenon is discussed in details elsewhere [2]. Nevertheless, it is clear from the voltammograms in Fig. 4 that lowering the temperature in the system leads to an increase of its electrochemical reversibility.

In Fig. 5A and B, we simulated the temperature influence on the peak currents and the half-peak widths of the net theoretical voltammograms. Similarly to the region of irreversible electron transfer, the net SW peak currents rise exponentially with decreasing temperature (Fig. 5A). The magnitudes of the half-peak widths are again a linear function of the temperature, but the slopes are basically independent of $\alpha$ (Fig. 5B). The independence of the slope $\Delta E_{\mathrm{p} / 2}-T$ on $\alpha$ distinguishes the quasireversible electron transfer from irreversible electron transfer (compare Figs. 5B and 3). At this stage, it is worth mentioning that for a given temperature, the half-peak widths of theoretical square-wave voltammograms are linear functions of the logarithm of the dimensionless kinetic parameter $K$ (in the region $-2<\log (K)<0.5$ ). This property is considered to be very useful for the determination of the standard rate constant of electron transfer, and it is discussed in detail in our concurrent paper (submitted).

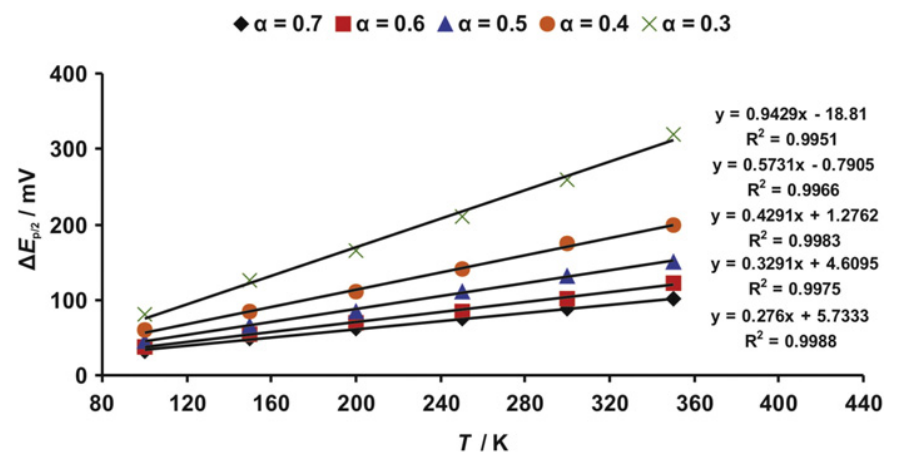

Fig. 3. Slow electron transfer: temperature dependence of the net SW half-peak widths simulated for various electron transfer coefficients. The other simulation details are same as those in Fig. 1. 

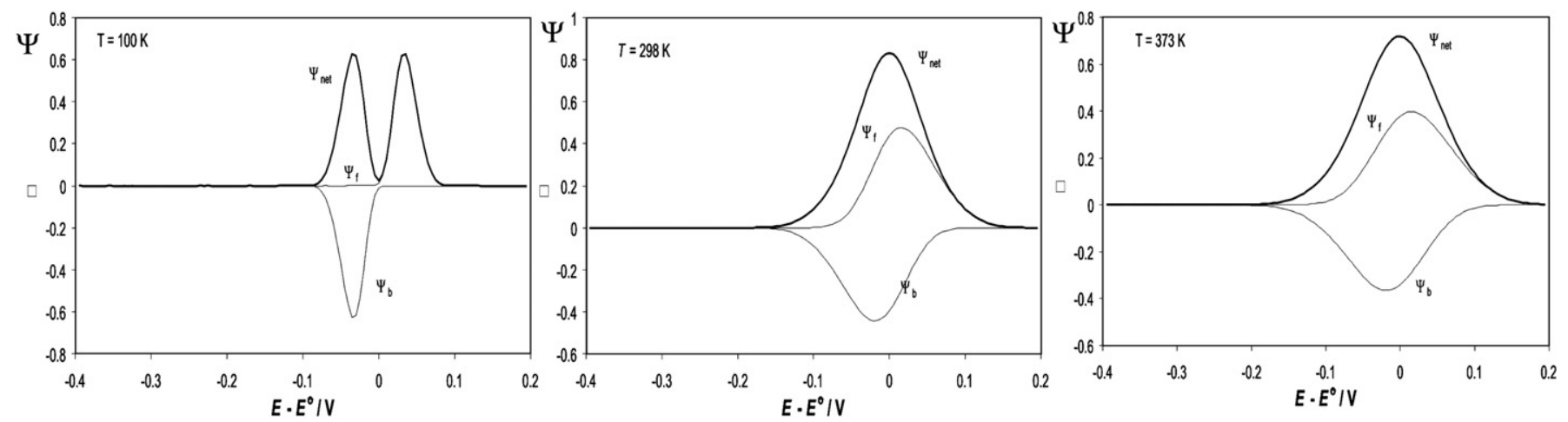

Fig. 4. Quasireversible electron transfer: square-wave voltammograms simulated for different temperatures. The simulation parameters are: dimensionless kinetic parameter $K=0.912$, square-wave amplitude $E_{\mathrm{sw}}=50 \mathrm{mV}$; potential increment $\Delta E=2 \mathrm{mV}$, and electron transfer coefficient $\alpha=0.5$.

The most remarkable attribute of the simple surface redox reaction studied under conditions of square-wave voltammetry is the parabolic dependence of the dimensionless peak current on the magnitude of the kinetic parameter $\log (K)[2,20]$. This feature is known as "quasireversible maximum", and it has been widely explored for estimation of the kinetics constant of electron transfer of various redox systems $[5,20,22]$. The redox reactions featuring moderate electron transfer (i.e. the quasireversible ones) usually give responses that are many times larger than much faster (reversible) reactions. This is mainly a consequence of the current sampling procedure that is used in pulse voltammetric techniques, but can also be attributed to the specific chronoamperometric properties of the surface redox reaction [2]. In Fig. 6, we simulated the dependence of the SWV net peak currents on $\log (K)$ for different temperatures. Generally, the position of the maximum is a function of the temperature, shifting towards lower values of $K$ with decreasing $T$. Up to temperatures of approximately $350 \mathrm{~K}$, there is a linear dependence between the $\log (K)$ and the temperature corresponding to the maximums of the curves in Fig. 6 (see inset in Fig. 6). The results presented in Fig. 6 demonstrate
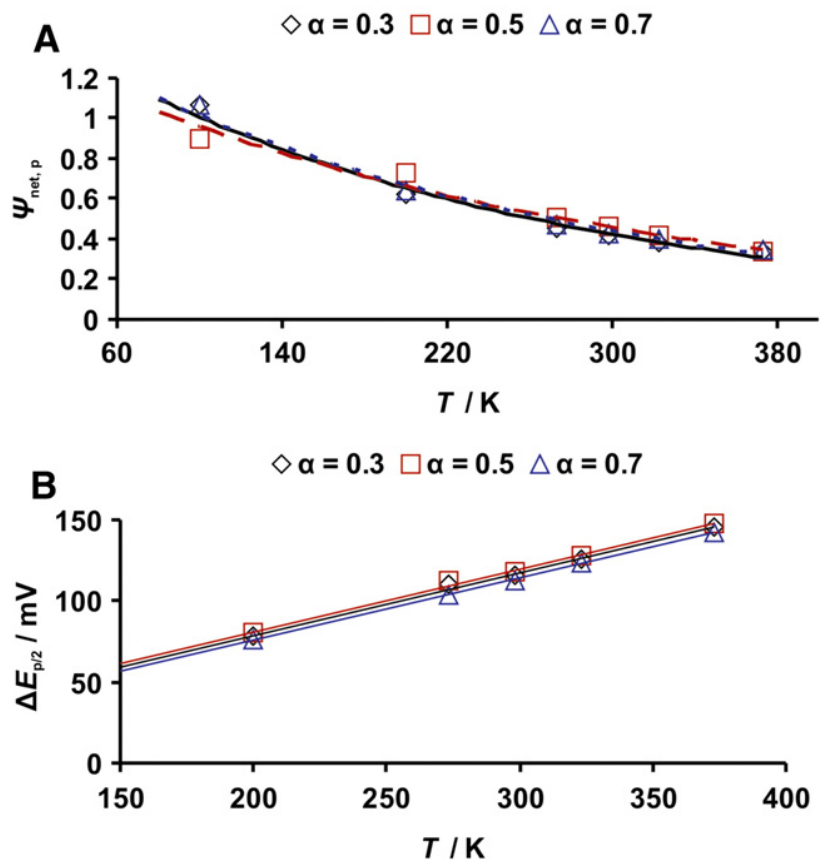

Fig. 5. Quasireversible electron transfer: A) The peak currents and B) the half-peak width temperature dependence of the theoretical square-wave voltammograms are shown. The value of the dimensionless kinetic parameter was $K=0.251$. The other simulation details are the same as those in Fig. 4. that variation of the temperature in the electrochemical cell can considerably change the reversibility of the redox system.

\subsection{Very fast electron transfer}

One of the most interesting items of the surface redox reaction of a strongly adsorbed redox couple is the "splitting of the net SW response" [19,23]. Commonly, for the values of the dimensionless parameter $\log (K)>0.6$ (i.e. very fast electron transfer) the net SW peak splits into two peaks that are symmetrically positioned around the standard redox potential. The large separation of the forward and backward current components is a consequence of the big values of the dimensionless rate constants of the oxidation and reduction halfreactions and the specific way of current sampling in square-wave voltammetry $[2,19,23]$. Experimentally, the splitting phenomenon can be achieved by decreasing the square-wave frequency or by increasing the SW amplitude [2,23]. The potential separation between the separated peaks is a function of the dimensionless kinetic parameter $K$, but also of the square-wave amplitude $E_{\mathrm{sw}}$. The features of the splitting phenomenon are discussed in detail elsewhere [23], and a very simple and powerful methodology for complete thermodynamic and kinetic characterization of surface redox reactions is available $[2,23]$. The effect of the temperature on the SW voltammograms featuring fast electron transfer is shown in Fig. 7. By decreasing the temperature from 298 to $100 \mathrm{~K}$, a slight increase in the potential separation between the split SW peaks can be observed. The width of the twin peaks is, however, very sensitive to the temperature changes,

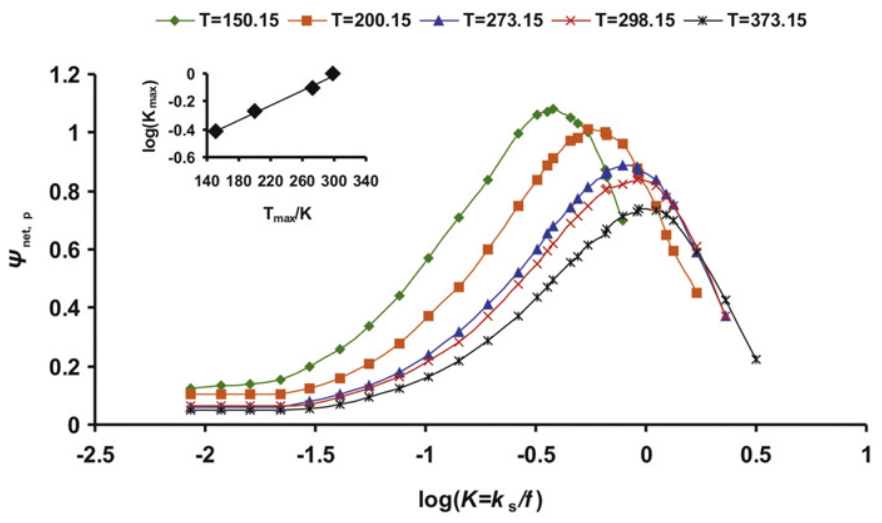

Fig. 6. Quasireversible electron transfer: influence of the temperature to the shape and position of the "quasireversible maximums". The inset shows the dependence between the critical values of $\log (K)$ and the temperatures corresponding to the quasireversible maximums. Square-wave amplitude $E_{\mathrm{sw}}=50 \mathrm{mV}$; potential increment $\Delta E=2 \mathrm{mV}$, and electron transfer coefficient $\alpha=0.5$. 

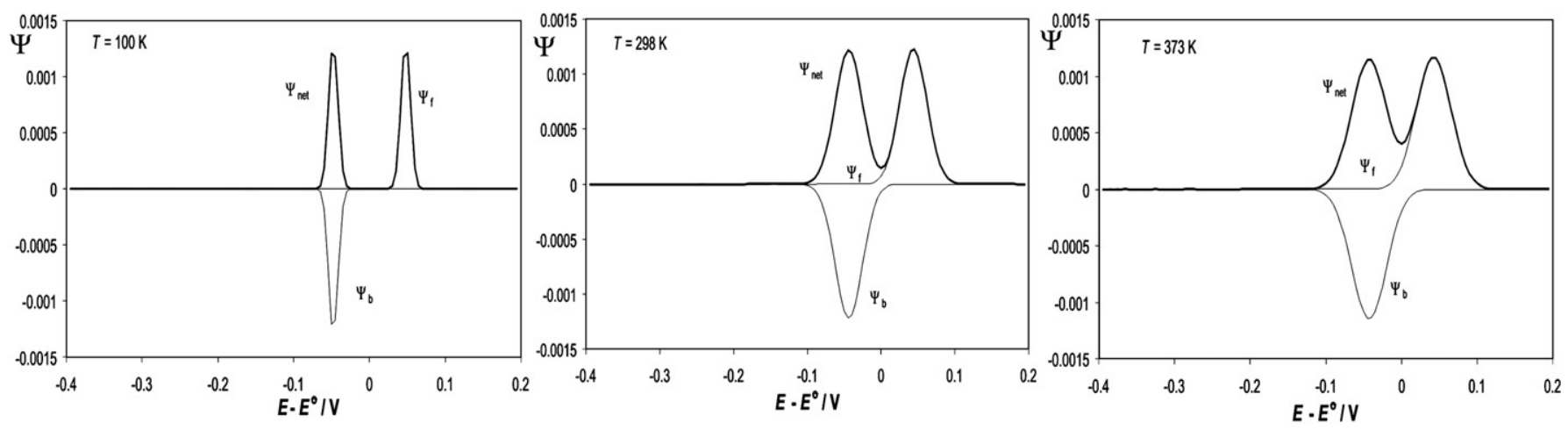

Fig. 7. Fast electron transfer: square-wave voltammograms simulated for different temperatures. The simulation parameters are: dimensionless kinetic parameter $K=11$, square-wave amplitude $E_{\mathrm{sw}}=50 \mathrm{mV}$; potential increment $\Delta E=2 \mathrm{mV}$, and electron transfer coefficient $\alpha=0.5$.

getting much narrower by decreasing the temperature. At very low temperatures, the twin peaks are finally seen as narrow spikes. This is a very specific voltammetric situation, and it can sometimes lead to wrong conclusions, especially if the properties of the surface redox reactions under conditions of square-wave voltammetry are not well known. The features of the SW voltammograms presented in Fig. 7 should avoid the dangers of invoking multiple species, "stable radicals", or additional processes to explain "odd" features met in some studies, such as appearance of multiple peaks or very narrow peaks [24-33], particularly in SW voltammetric studies performed under cryogenic conditions.

3.4. Analysis of the temperature effect relevant to the study of a single electrode reaction

The voltammetric response of a single electrode reaction is an item of great interest. Therefore, we have analyzed its temperature dependence. As mentioned earlier, Eq. (9) explicitly shows that the temperature affects the voltammetric responses through two parameters, i.e., the relative dimensionless potential $\phi$ and the dimensionless electrode kinetic parameter $K$, which are altered simultaneously by varying $T$. The temperature effect on the value of the standard rate constant of electron transfer is commonly represented in the Arrhenius form, i.e. $k_{\mathrm{s}}=A \exp$ $\left(-E_{\mathrm{a}}(R T)^{-1}\right)$, where $A\left(\mathrm{~s}^{-1}\right)$ is the frequency factor of the electron transfer reaction, while $E_{\mathrm{a}}\left(\mathrm{J} \mathrm{mol}^{-1}\right)$ is the electron transfer activation energy [3]. The theoretical calculations performed under "Arrhenius" conditions show that the temperature effect on the features of the simulated square-wave voltammograms is identical to that of the dimensionless kinetic parameter $K$ [2]. The new item observed in this situation is the option to affect the properties of the "quasireversible maximum" additionally by varying the temperature. In Fig. 8A, several "quasireversible maximums" were calculated for four different temperatures under "Arrhenius" conditions. Note that the "quasireversible maximums" in Fig. 8A are calculated by altering the SW frequency, which is a usual analysis when performing real experiments. An increase of the temperature is paralleled by a shift of the position of the "quasireversible maximums" towards lower frequency values (i.e. toward higher values of the kinetic parameter $K$ ). For these simulations, we used the following values: $E_{\mathrm{a}}=20,000 \mathrm{~J} \mathrm{~mol}^{-1}$, and $A=10^{6} \mathrm{~s}^{-1}$. The values for $E_{\mathrm{a}}$ and $A$ are taken from reference [34] and they are a result of electron transfer kinetics studies at a graphite electrode-protein interface. A very important result of the last figure is the linear dependence between the logarithm of the values of the critical frequencies (corresponding to the maximums of the parabolic curves $-\log \left(f_{\text {max }}\right)$ ) and the inverse values of the temperatures $1 / T$ (see Fig. 8B). The linear dependence between log $\left(f_{\max }\right)$ and $1 / T$ has the following form:

$\log \left(f_{\max }\right)=-934.02 T^{-1}+5.6733$
It is important to note, that for a given temperature, the "quasireversible maximum" appears when the SW frequency of the signal fulfils the condition: $K_{\max }=A f_{\max }^{-1} \exp \left[-E_{\mathrm{a}}(\mathrm{RT})^{-1}\right]$. From this expression, it follows that the maximal SW frequency $f_{\max }$, at which the "quasireversible maximum" appears, is given by:

$\ln \left(f_{\max }\right)=\ln (A)-\ln \left(K_{\max }\right)-\left(E_{a} R^{-1}\right) T^{-1}$

The last equation is of great importance, since it permits to determine the frequency factor of the electron transfer reaction $A$

A

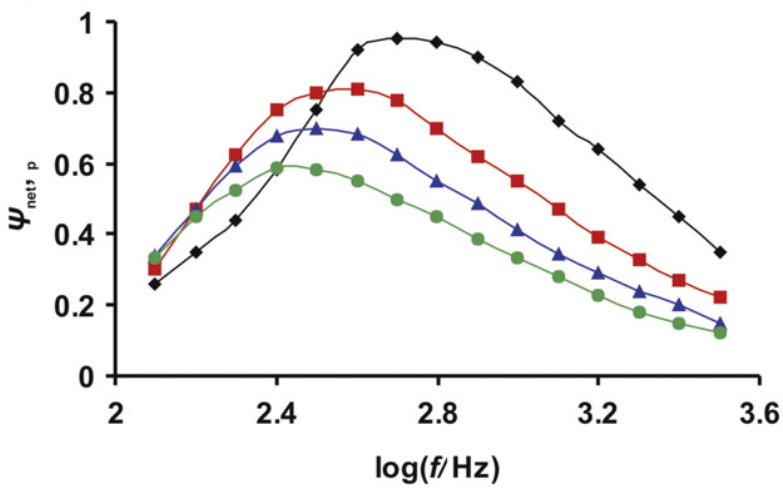

B

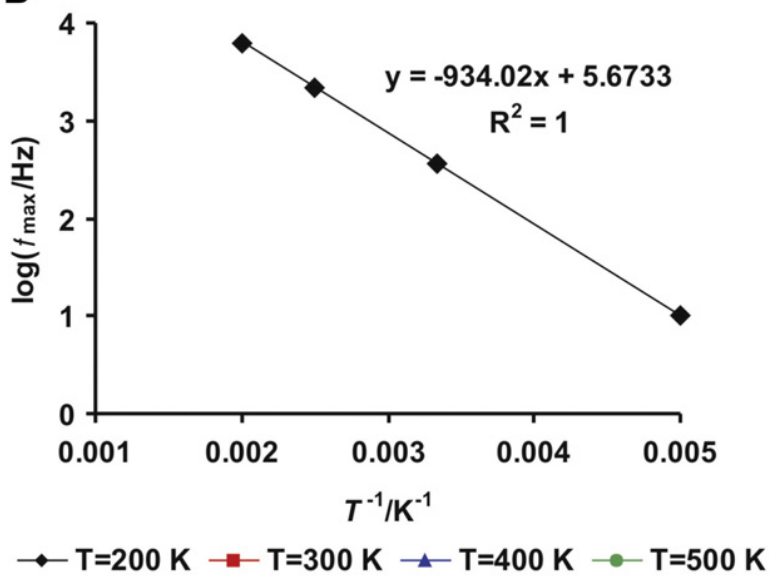

Fig. 8. A) Temperature effect to the theoretical "quasireversible maximums" simulated under "Arrhenius" conditions. B) shows the dependence between the logarithm of the values of the frequencies corresponding to the maximums of the parabolic curves-log $\left(f_{\max }\right)$ and the inverse values of the temperatures $1 / T$. For these calculations, the following parameters have been used: square-wave amplitude $E_{\mathrm{sw}}=50 \mathrm{mV}$; potential increment $\Delta E=2 \mathrm{mV}$, number of exchanged electrons $n=1$, electron transfer coefficient $\alpha=0.5$, activation energy $E_{\mathrm{a}}=20,000 \mathrm{~J} \mathrm{~mol}^{-1}$, and frequency factor $A=10^{6} \mathrm{~s}^{-1}$. 
(from the intercept of the dependence $\left.\ln \left(f_{\max }\right)--T^{-1}\right)$, as well as the electron transfer activation energy $E_{\mathrm{a}}$ (from the slope of the dependence $\left.\ln \left(f_{\max }\right)-T^{-1}\right)$. These are, indeed, the crucial physical parameters characterizing the thermodynamics of the electron transfer step. The value of $\log \left(K_{\max }\right)$ at a given temperature can be estimated from the linear dependence of $\log \left(K_{\max }\right)$ and $T$ (inset of Fig. 6), which is:

$\log \left(K_{\max }\right)=0.0027 T-0.82$

In one practical example, we show how the thermodynamic parameters of the electron transfer reaction can be estimated with this methodology. By exploring the value of $\log \left(K_{\max }\right)=0$ at $T=300 \mathrm{~K}$ (see inset from Fig. 6), with the help of the equation of the linear dependence $\log \left(f_{\max }\right)-T^{-1}$ (Eqs. (10) and (11)), we estimated the values of $E_{\mathrm{a}}$ and $A$ being $17,900 \mathrm{~J} \mathrm{~mol}^{-1}$ and $5.7 \times 10^{5} \mathrm{~s}^{-1}$, respectively. The values of $E_{\mathrm{a}}$ and $A$ have been identified from the Eq. (11). These estimated values for $E_{\mathrm{a}}$ and $A$ are in very good agreement with the values used for the simulations of Fig. $8\left(E_{\mathrm{a}}=20,000 \mathrm{~J} \mathrm{~mol}^{-1}\right.$, and $A=10^{6} \mathrm{~s}^{-1}$ ), a fact that shows the reliability of the proposed methodology. It should be also mentioned that the electron transfer activation energy $E_{\mathrm{a}}$ is closely connected to the standard redox potential of the investigated redox compounds [3]. The last Fig. 8A and $B$ show that the temperature effect on the phenomenon of "quasireversible maximum" can be viewed as a simple and viable way to precisely estimate the thermodynamic parameters as the standard redox potentials of lipophilic proteins and other surfaceactive redox compounds.

\section{Conclusions}

In this paper we analyzed the effect of the temperature on theoretical square-wave voltammograms of a surface redox reaction, which is considered as an adequate model of protein-film voltammetric experiments. Protein-film voltammetry is a relatively new concept, which offers many interesting opportunities for fundamental and applied research. Being extensively explored to investigate the kinetics of electron transfer and protein-protein interactions in various scenarios, the protein-film voltammetry appears to be a very simple and efficient tool to understand the redox properties of various enzymes.

Electron transfer reactions are central to the function of proteins in many biological processes. This is well known in bioenergetics: photosynthesis and respiration realize energy conversion through a complex sequence of electron transfer reactions. However, electron transfer also takes place in many other biological processes ranging from cell defense to gene control. The rate of electron transfer from a donor $\mathrm{D}$ to an acceptor $\mathrm{A}$ is a key parameter that determines biological function, and much effort has been made to relate the rate of electron transfer to structural and thermodynamic features of the compounds of interest. In the last two decades, square-wave voltammetry has emerged as one of the leading voltammetric techniques in respect of the kinetics characterization of chemical and electron transfer steps by various surface electrode reactions [2,35]. It offers relative simple modes for recognition of the electrode mechanisms, as well as for measuring their kinetics [2,35-41].

We have analyzed temperature effects to the protein-film squarewave voltammetric responses featuring slow, quasireversible and fast electron transfer. Generally, by decreasing the temperature of the system, an increase of the electrochemical reversibility of the surface redox systems has been observed. In the region of very slow electron transfer, the magnitude of the half-peak width of the theoretical SW voltammograms decreases linearly with decreasing the temperature. The slope of half-peak width against $T$ was found to be inversely proportional to the electron transfer coefficient $\alpha$. The slope $\Delta E_{\mathrm{p} / 2} v s$. $T$ was, however, independent on the kinetic parameter $K$ if the value of
$K$ was $\leq 0.005$. This means that the linear dependence between $\Delta E_{\mathrm{p} / 2}$ and $T$ in the irreversible region (defined as $\Delta E_{\mathrm{p} / 2}=\left(z \frac{R}{\alpha n F}\right) T$, where the factor $z$ in the slope is a constant depending on $\left.E_{\mathrm{sw}}\right)$ can be used to estimate the electron transfer coefficient $\alpha$.

Lowering the temperature significantly affects the phenomena of the "quasireversible maximum" and "splitting SW peaks", which are the main kinetic attributes of the surface redox reactions featuring moderate and fast electron transfers [2]. The appearance of the spiky twin peaks by surface redox systems with very fast electron transfer at low temperatures should not be interpreted as an abnormal feature of square-wave voltammetry. Since the surface redox reaction is considered as an adequate model for a protein-film voltammetric experiments, our simulations should help to elucidate the redox mechanisms and to determine the kinetic parameters of protein-film criovoltammetry. Moreover, by simulating the temperature effects of the "quasireversible maximum" under "Arrhenius" conditions, i.e. by considering the temperature effects of the standard rate constant of a single electrode reaction, we have shown how elegantly one can calculate important thermodynamic parameters of the surfaceconfined electron transfer reactions. We give in this work a theoretical equation (Eq. (12)) from which one can calculate the critical value of the kinetic parameter corresponding to the quasireversible maximum $K_{\max }$ at a given temperature. In the real experiment, the quasireversible maximums presented in Fig. 8A could be demonstrated by varying the SW signal frequency. Plotting the ratio of the real peak current and the corresponding frequency $-I_{\mathrm{p}} /$ f versus the logarithm of the signal frequency $\log (f)$ at several temperatures, one could reconstruct the theoretical dependences depicted in Fig. 8A. If the critical frequencies associated with the quasireversible maximums $f_{\max }$ are obtained experimentally from the parabolic curves $I_{\mathrm{p}} / f v s . \log$ $(f)$, and if the critical values of the kinetic parameter $K_{\max }$ at a given temperature are calculated theoretically by using Eq. (12), then, by plotting the dependence between the critical values of the estimated frequencies $\log \left(f_{\max }\right) v s .1 / T$, one should get a linear regression line as presented in Fig. 8B. The slope of this linear dependence allows to obtain the value of the activation energy of electron transfer $E_{\mathrm{a}}$, while the frequency factor of the electron transfer reaction $A$ can be determined from the interception (see Eq. (11)). To the best of our knowledge, this is the first theoretical work under conditions of SWV, which shows that the "quasireversible maximum" can be explored to determine the activation energy of electron transfer reactions in surface redox systems, and consequently, for the determination of the standard redox potential of many surface-active compounds. These calculations unanimously show that the phenomenon of "quasireversible maximum" can be explored simultaneously for both, the kinetic and the thermodynamic characterization of the electron transfer steps during surface redox reactions.

\section{Acknowledgments}

Rubin Gulaboski thanks Alexander von Humboldt Stiftung for providing a postdoctoral fellowship.

This project was funded by the Deutsche Forschungsgemeinschaft (SFB 530, project A3, and the Graduate Colleges GK1276 and GK845, all to $M$. Hoth) and two competitive research grants from the Saarland University (HOMFOR both to I. Bogeski).

\section{References}

[1] M.R. Smyth, J.G. Vos, Analytical Voltammetry in Comprehensive Analytical Chemistry, Elsevier, New York, 1992.

[2] V. Mirceski, S. Komorsky-Lovric, M. Lovric, Square-Wave Voltammetry, Theory and Application, Springer, Berlin, 2007.

[3] A.J. Bard, L.R. Faulkner, Electrochemical Methods, Fundamentals and Applications, John Wiley \& Sons, New York, 2001.

[4] R.J. Dwayne Miller, G. McLendon, A.J. Nozik, A. Schmickler, F. Willig, Surface Electron Transfer Processes, Wiley, New York, 1995

[5] F.A. Armstrong, Bioelectrochemistry of Biomacromolecules, Birkhauser, Basel, 1997. 
[6] F.A. Armstrong, H.A. Heering, J. Hirst, Reactions of complex metalloproteins studied by protein-film voltammetry, Chem. Soc. Rev. 26 (1997) 169-179.

[7] S.E.J. Fawcett, D. Davis, J.L. Breton, A.J. Thomson, F.A. Armstrong, Voltammetric studies of the reactions of iron-sulphur clusters ([3Fe-4S] or [M3Fe-4S]) formed in Pyrococcus furiosus ferredoxin, Biochem. J. 335 (1998) 357-368.

[8] C. Leger, S.J. Elliott, K.R. Hoke, L.J.C. Jeuken, A.K. Jones, F.A. Armstrong, Enzyme electrokinetics: using protein film voltammetry to investigate redox enzymes and their mechanisms, Biochemistry (Us) 42 (2003) 8653-8662.

[9] J.P. McEvoy, F.A. Armstrong, Protein film cryovoltammetry: demonstrations with a 7Fe ([3Fe-4S]+[4Fe-4S]) ferredoxin, Chem. Commun. (1999) 1635-1636.

[10] P. Obrien, D.A. Sweigart, Low-temperature electrochemistry of chromium porphyrins-characterization of transient species, J. Chem. Soc. Chem. Comm. (1986) 198-200.

[11] G.K. Rowe, M.T. Carter, J.N. Richardson, R.W. Murray, Consequences of kinetic dispersion on the electrochemistry of an adsorbed redox-active monolayer, Langmuir 11 (1995) 1797-1806.

[12] J.N. Richardson, S.R. Peck, L.S. Curtin, L.M. Tender, R.H. Terrill, M.T. Carter, R.W. Murray, G.K. Rowe, S.E. Creager, Electron-transfer kinetics of self-assembled ferrocene octanethiol monolayers on gold and silver electrodes from 115-K to 170-K, J. Phys. Chem. (Us.) 99 (1995) 766-772.

[13] M.S. Ravenscroft, H.O. Finklea, Kinetics of electron-transfer to attached redox centers on gold electrodes in nonaqueous electrolytes, J. Phys. Chem. (Us.) 98 (1994) 3843-3850.

[14] C.Y. Yao, T.H. Kao, C.H. Cheng, J.M. Chen, W.M. Hurng, Studies of electrochemical properties of lithium cobalt oxide, J. Power Sources 54 (1995) 491-493.

[15] A. El Kasmi, J.M. Wallace, E.F. Bowden, S.M. Binet, R.J. Linderman, Controlling interfacial electron-transfer kinetics of cytochrome c with mixed self-assembled monolayers, J. Am. Chem. Soc. 120 (1998) 225-226.

[16] J.L. Kong, Z.O. Lu, Y.M. Lvov, R.Z.B. Desamero, H.A. Frank, J.F. Rusling, Direct electrochemistry of cofactor redox sites in a bacterial photosynthetic reaction center protein, J. Am. Chem. Soc. 120 (1998) 7371-7372.

[17] J. Hirst, F.A. Armstrong, Fast-scan cyclic voltammetry of protein films on pyrolytic graphite edge electrodes: characteristics of electron exchange, Anal. Chem. 70 (1998) 5062-5071.

[18] R.S. Nicholson, M.L. Olmstead, in: J.S. Mattson, H.B. Mark, H.C. MacDonald (Eds.), Electrochemistry: Calculations, Simulation and Instrumentation, Marcel Dekker, New York, 1972, pp. 120-137.

[19] J.J. Odea, J.G. Osteryoung, Characterization of quasi-reversible surface processes by square-wave voltammetry, Anal. Chem. 65 (1993) 3090-3097.

[20] S. Komorsky-Lovric, M. Lovric, Kinetic measurements of a surface-confined redox reaction, Anal. Chim. Acta. 305 (1995) 248-255.

[21] S. Komorsky-Lovric, M. Lovric, Square-wave voltammetry of quasi-reversible surface redox reactions, J. Electroanal. Chem. 384 (1995) 115-122.

[22] M. Lovric, A quasi-reversible maximum in square-wave voltammetry, Sov. Electrochem. 27 (1991) 168-173.

[23] V. Mirceski, M. Lovric, Split square-wave voltammograms of surface redox reactions, Electroanal 9 (1997) 1283-1287.

[24] J.A. Mertens, N. Shiraishi, W.H. Campbell, Recombinant expression of molybdenum reductase fragments of plant nitrate reductase at high levels in Pichia pastoris, Plant Physiol. 123 (2000) 743-756.
[25] K.C. Kemp, E. Fourie, J. Conradie, J.C. Swarts, Ruthenocene-containing betadiketones: synthesis, pK(a)' values, keto-enol isomerization kinetics, and electrochemical aspects, Organometallics 27 (2008) 353-362.

[26] F.Y. Wang, A. Tessier, J. Buffle, Voltammetric determination of elemental sulfur in pore waters, Limnol. Oceanogr. 43 (1998) 1353-1361.

[27] E.M. Ghoneim, Electroreduction of the muscle relaxant drug dantrolene sodium at the mercury electrode and its determination in bulk form and pharmaceutical formulation, Chem. Pharm. Bull. 55 (2007) 1483-1488.

[28] P.E.M. Phillips, R.M. Wightman, Critical guidelines for validation of the selectivity of in-vivo chemical microsensors, TrAC, Trends Anal. Chem. 22 (2003) 509-514.

[29] N. Serrano, J.M. Diaz-Cruz, C. Arino, M. Esteban, Comparison of constant-current stripping chronopotentiometry and anodic stripping voltammetry in metal speciation studies using mercury drop and film electrodes, J. Electroanal. Chem. 560 (2003) 105-116.

[30] N. Serrano, I. Sestakova, J.M. Diaz-Cruz Constant current strippingchronopotentiometry for the study of adsorbing inert and electrochemically nonreversible metal complexes at low concentrations: application to $\mathrm{Cd}$ and $\mathrm{Zn}$ metallothioneins, Electroanalysis 18 (2006) 169-176.

[31] S.E. Creager, G.T. Marks, D.A. Aikens, H.H. Richtol, Linear sweep voltammetry of adsorbed neutral red, J. Electroanal. Chem. 152 (1983) 197-209.

[32] C.C. Hardin, J.L. Sneeden, S.M. Lemon, B.A. Brown, R.H. Guenther, H. Sierzputowska-Gracz, Folding of pyrimidine-enriched RNA fragments from the vicinity of the internal ribosomal entry site of Hepatitis A virus, Nucleic Acids Res. 27 (1999) 665-673.

[33] A. Evans, M.I. Montenegro, D. Pletcher, The mechanism for the cathodic reduction of sulphur in dimethylformamide: low temperature voltammetry, Electrochem. Commun. 3 (2001) 514-518.

[34] L.J.C. Jeuken, J.P. McEvoy, F.A. Armstrong, Insights into gated electron-transfer kinetics at the electrode-protein interface: a square wave voltammetry study of the blue copper protein azurin, J. Phys. Chem. B 106 (2002) 2304-2313.

[35] J. Osteryoung, J.J. O’Dea, Square-wave voltammetry, in: A.J. Bard (Ed.), Electroanalytical chemistry, vol. 14, Marcel Dekker, New York, 1986, pp. 209-325.

[36] A.B. Miles, R.G. Compton, The theory of square wave voltammetry at uniformly accessible hydrodynamic electrodes, J. Electroanal. Chem. 487 (2000) 75-89.

[37] A.B. Miles, R.G. Compton, Simulation of square-wave voltammetry at a channel electrode: E, EC and ECE processes, J. Electroanal. Chem. 499 (2001) 1-16.

[38] B.A. Brookes, R.G. Compton, Simulation of square wave voltammetry: quasireversible electrode processes, J. Phys. Chem. B 103 (1999) 9020-9028.

[39] B.A. Brookes, J.C. Ball, R.G. Compton, Simulation of square wave voltammetry: reversible electrode processes, J. Phys. Chem. B 103 (1999) 5289-5295.

[40] B.A. Brookes, G. Macfie, R.G. Compton, Simulation of square wave voltammetry at hemispherical electrodes: electrochemically reversible, irreversible and quasireversible processes, J. Phys. Chem. B 104 (2000) 5784-5789.

[41] H. Shen, J.E. Mark, C.J. Seliskar, H.B. Mark Jr., W.R. Heineman, Blocking behavior of self-assembled monolayers on gold electrodes, J. Solid. State. Electrochem. 1 (1997) $148-154$. 